\title{
Electrocardiographic Recording with Conformable Organic Electrochemical Transistor Fabricated on Resorbable Bioscaffold
}

Alessandra Campana, Tobias Cramer, Daniel Simon, Magnus Berggren and Fabio Biscarini

\author{
Linköping University Post Print
}

\section{Tweet}

N.B.: When citing this work, cite the original article.

Original Publication:

Alessandra Campana, Tobias Cramer, Daniel Simon, Magnus Berggren and Fabio Biscarini, Electrocardiographic Recording with Conformable Organic Electrochemical Transistor Fabricated on Resorbable Bioscaffold, 2014, Advanced Materials, (26), 23, 3874-3878.

http://dx.doi.org/10.1002/adma.201400263

Copyright: Wiley-VCH Verlag

http://www.wiley-vch.de/publish/en/

Postprint available at: Linköping University Electronic Press

http://urn.kb.se/resolve?urn=urn:nbn:se:liu:diva-109181 
DOI: $10.1002 /(($ please add manuscript number $))$

Article type: Communication

\section{Electrocardiographic Recording with Conformable Organic Electrochemical Transistor Fabricated on Resorbable Bioscaffold}

Alessandra Campana, Tobias Cramer*, Daniel T. Simon, Magnus Berggren, Fabio Biscarini*

A. Campana, Dr. T. Cramer, Prof. F. Biscarini

Consiglio Nazionale delle Ricerche, Istituto per lo Studio dei Materiali Nanostrutturati (CNRISMN)

Via P. Gobetti 101

40129 Bologna, Italy

t.cramer@bo.ismn.cnr.it

A. Campana

Alma Mater Studiorum-Università degli Studi di Bologna, Dipartimento di Chimica "G.

Ciamician"

Via F. Selmi 2

40127 Bologna, Italy

Dr. D. T. Simon, Prof. M. Berggren

Department of Science and Technology, Linköping University

SE-601 74 Norrköping, Sweden

Prof. F. Biscarini

Life Science Dept., Università di Modena e Reggio Emilia

Via Campi 183

41125 Modena, Italy

fabio.biscarini@unimore.it

Keywords: organic electronics, organic transistors, bioelectronics, biomedical applications

Electrical impulses accompany many of our bodies daily activities ranging from thought, muscular activity to vegetative processes like digestion. Abnormalities in these bioelectric signals are related to severe diseases for which modern medicine is developing treatments. As an alternative or complement to pharmaceuticals, modern medicine has started to develop a more technological approach which is based on electrical devices (termed electroceuticals ${ }^{[1]}$ ) which interface directly to muscular tissue, nerve fibers or brain circuits with the possibility of diagnosing and treating an array of conditions. Cardiac diseases have already become a well-established area for the application of electrical devices; they are detected by electrocardiographic (ECG) recordings and real-time 
treatment can be provided by implanted pacemakers. Other pathological conditions where malfunctioning of the electrical signal propagation could be addressed with electroceuticals include some neurodegenerative diseases (e.g. Parkinson's) and spinal cord injury. ${ }^{[2]}$ In order to further develop electroceutical treatments, new generations of implantable devices have to be developed which combine the possibility to interface with bioelectric activity while at the same time minimizing the invasiveness of the device during implantation, operation and possibly removal.

Material science can provide a major contribution to this quest as the invasiveness of devices is, to a large extent, controlled by the properties of the materials and their surfaces. ${ }^{[3]}$ In particular, a material platform has to be defined which confers flexibility and adaptability in order to allow fabrication of devices with mechanical properties in compliance with soft biological tissue. ${ }^{[4]}$ Another desirable feature would be the resorption of the employed materials by the human body. ${ }^{[5]}$ Today, a palette of polymers and biopolymers are known and utilized, for example in drug release or orthopaedic implants, which decompose on a controlled timescale when incorporated in the body. Examples are natural polypeptides like silk fibroin ${ }^{[6]}$ or synthetic polyesters like poly(Llactide-co-glycolide) (PLGA) $)^{[7,8]}$, which represents the most widely used material class for temporary implants. Bioresorption is advantageous for implants as they become transient objects in the body, making implant removal unnecessary and also minimizing the occurrence of the systemic reaction to foreign bodies. Within this context, organic as well as inorganic materials platforms have been proposed which allow for the fabrication of electronic devices whose components degrade into soluble, nontoxic fragments on a controlled timescale in the human body or other biological environments. ${ }^{[9-11]}$

With the goal to pave the way for low-invasive electroceutical treatments, we report here a simple fabrication process which allows to pattern a bioelectronic interface on a bioresorbable scaffold. The resulting prototype device combines optimized materials properties for implantation such as 
flexibility, transparency and bioresorption with excellent potentiometric recording capabilities. We demonstrate the functionality of the bioelectronic interface by performing ECG recordings. As active electronic material we process the conducting polymer mixture PEDOT:PSS as employed in organic electrochemical transistors (OECTs).

In recent years various material platforms have been employed to fabricate devices for bioelectric recordings and stimulations. ${ }^{[12]}$ Organic electronics provides conductors and semiconductors which are particular advantageous for biomedical implants as they can be processed into ultra-light, soft and flexible electronic devices. ${ }^{[13,14]}$ In addition, organic materials can be chemically designed to retain their electrical properties even when immersed in aqueous medium. ${ }^{[15,16]}$ In this way, a low impedance interface between ionic signals in solution and electronic signals in the device can be created. Sophisticated architectures for bioelectric recordings rely on the intrinsic amplification properties of transistors as potentiometric sensors. ${ }^{[1-19]}$ In organic transistors a current is driven through a thin layer of organic semiconductor by applying a potential difference to two electrical contacts termed source and drain. The source-drain current, $I_{S D}$, is highly sensitive to the electric field originating from a third electrode, the gate. As the gate is capacitively coupled to the semiconducting channel, changes in its potential lead to accumulation or depletion of charge carriers in the channel, with a consequent variation of the monitored $I_{S D}$. The effect is exploited in bioelectric recordings by gating the channel with an aqueous electrolyte that contains the biological system. Due to the activity of electrogenic cells like neurons or cardiomyocites, potential variations in the range of some ten to hundreds of microvolts emerge and leave their amplified trace in $I_{S D}$. Recently, PEDOT:PSS-based OECTs fabricated in micrometer sized arrays on a parylene substrate have shown superior performance with respect to standard potentiometric measurements for in vivo recordings of brain activity. ${ }^{[19]}$ PEDOT:PSS provides larger transconductance than field effect devices $^{[20]}$ as ions from the liquid film can migrate into the swelling polymer where they induce reversible depletion of charge carriers via redox reactions on the PEDOT. ${ }^{[21]}$ As a consequence, 
potential changes in the liquid modulate the carrier density in the whole volume of the PEDOT:PSS film, thereby increasing the interfacial capacitance an additional order of magnitude and making the material a convenient choice for optimized ion-electron exchange. ${ }^{[21-23]}$

Fabrication of the OECT on the PLGA bioscaffold has to overcome two criticalities: First, microstructuring of PEDOT:PSS has to be achieved without solvent based lithographic procedures as PLGA is highly soluble. ${ }^{[24]}$ Second, surface chemistry has to be optimized to avoid delamination of the swellable PEDOT:PSS. Figure 1a depicts a schematic drawing of the device architecture. The $20 \mu \mathrm{m}$ thick homogeneous and transparent PLGA substrate was prepared by solvent casting (5\% wt solution in chloroform). By tightly controlling environmental conditions, films could be prepared with an averaged mean roughness of $2 \mathrm{~nm}$ as determined by AFM measurements (Supp. Inf. S1). Bioresorption of the PLGA scaffold depends on ambient conditions like $\mathrm{pH}$ or temperature ${ }^{[25]}$ as well as the composition in terms of the monomeric ratio. In this case 75:15 D,L-lactide:glycolide units sets the half-life time scale in the range of weeks to months under normal physiologic conditions. ${ }^{[8]}$ In our experimental setting, no visual degradation of the film could be observed on the days time scale at which measurements were undertaken. On top of the PLGA film metallic source and drain contacts were deposited by the evaporation of gold $(30 \mathrm{~nm})$ through a shadow mask $(\mathrm{L}=$ $30 \mu \mathrm{m}, \mathrm{W}=1000 \mu \mathrm{m})$. Next, PEDOT:PSS was spin coated on the substrate and thermally annealed $\left(50^{\circ} \mathrm{C}, 24 \mathrm{~h}\right)$ to form ca. $200 \mathrm{~nm}$ thick layers. The incorporation of an epoxy cross-linker and, more importantly, $\mathrm{pH}$ neutrality of the aqueous PEDOT:PSS suspension to avoid $\mathrm{pH}$-catalyzed hydrolysis of the scaffold and subsequent delamination were found to be crucial for adhesion on the PLGA scaffold. Patterning of PEDOT:PSS was achieved by a dry etching process $(20 \mathrm{~s}, 150 \mathrm{~W}, 600$ mTorr, $\mathrm{O}_{2}: \mathrm{CF}_{4}=1: 1$ ). Transistor regions were protected through pressing an elastomeric stamp (polydimethylsiloxane) on their surface. Figure 1b shows a micrograph of a transistor with a channel length of $30 \mathrm{um}$. The area of PEDOT:PSS is visual as a darker color of the gold electrodes. The photography in Figure 1c visualizes optical and mechanical properties of the device such as 
optical transparency and mechanical flexibility which confers adaptability to curved biologic tissues.

To characterize the electrochemical transistor, the channel region was contacted by aqueous phosphate buffer solution (0.1 M PBS, $\mathrm{pH}$ 7) in which a Pt or Ag wire was immersed and used as the gate electrode. Figure 2 shows characteristic output and transfer curves of fabricated devices. Stable low voltage operation is achieved as in state-of-the-art OECTs. ${ }^{[26]}$ As in normal thin film transistors the output curves in Figure 2a present linear behavior at low drain potentials while current saturation is observed when the drain potential is increased. As the transistor works in depletion mode, reduction of the transistor current occurs when setting the electrolyte to positive potential with respect to the PEDOT:PSS channel ${ }^{[27]}$. In this way mobile positive carriers in the $\mathrm{p}$ type semiconducting polymer PEDOT are reduced and cations from solutions migrate into the film to counterbalance the negative charges of the polyanionic dopant PSS. As charge modulation effects the whole volume of the film, the transistor shows a huge interfacial capacitance of $\sim 500 \mu \mathrm{F} \mathrm{cm}{ }^{-2}$ in the low frequency limit (Supp. Inf. S2) and a large transconductance of $3.2 \pm 0.5 \mathrm{mS}$ at $V_{S D}=-1 \mathrm{~V}$ as extracted from Fig 2 b. In these measurements Faradaic leakage currents remain more than three orders of magnitude below $I_{S D}$. Next, we tested the influence of conforming the device to nonplanar surfaces on the electrical performance. Fig $2 \mathrm{~b}$ illustrates a transfer measurement (dashed line) obtained after mechanically stressing the device by bending with radii down to $80 \mu \mathrm{m}$ (Supp. Inf. S3). The observed variations were small when compared to the standard variation in measurements of unstressed devices (grey areas in Figure 2b). Also, mechanically stressing the device by attaching it to curved biological surfaces like human skin (Figure 1c) did not result in device failure. Even in cases of extreme stressing like crumpling of the device with repeated pressing on folding edges, no dewetting or disruption of the polymeric layer could be observed, although devices did start to show failure due to cracks in the gold electrodes. In order to assess the devices as a potentiometric sensors we operated the transistor at constant gate and drain potential 
$\left(V_{S G}=0.3 \mathrm{~V} ; V_{S D}=-0.3 \mathrm{~V}\right)$ in electrolyte solution and sampled the drain current. Application of small potential pulses to the gate resulted in characteristic changes in drain current, depicted in Figure 2c. High sensitivity combined with operational stability is demonstrated by the clear detection of potential changes as low as $\Delta V_{S G}=50 \mu \mathrm{V}$ on seconds time scale. In order to define the response time of the device we applied a larger potential pulse $\left(\Delta V_{S G}=0.1 \mathrm{~V}\right)$ and sampled the current at a rate of $10 \mathrm{kHz}$. The resulting response is shown in Figure $2 \mathbf{d}$ and follows the exponential behavior of an RC circuit with a time constant of $\tau=1.5 \mathrm{~ms}$.

In order to demonstrate the potentiometric recording capabilities of the bioresorbable OECT in a medically relevant setting, we record a human ECG. The ECG signal originates in the muscular tissue of the heart where, at the cellular basis, muscle cell contraction is initiated by an influx of cations causing cell depolarization. The ordered progression of contraction throughout the heart muscle gives rise to a macroscopic ionic current wave that spreads throughout the body during each heart beat cycle leading to tiny potential changes on the skin due to the finite resistance of the body's tissue. By attaching electrodes, the signal can be recorded by a sensitive potentiometer. From the recorded shape one can extract information about the pulse rate, pulse regularity as well as heart dimensions and the presence of damage. Traditional $\mathrm{Ag} / \mathrm{AgCl}$ based electrodes establish a Faradaic contact to the $\operatorname{skin}^{[28]}$. In the case of an OECT based ECG sensor, the recording occurs by attaching the transistor's exposed PEDOT:PSS channel directly to the skin. We used electroconductive gel, as usual in ECG recordings, to promote adhesion and to reduce the skin's impedance. In that way, the skin replaces the role of the gate and its potential changes with respect to a ground contact lead to transient fluctuations of the drain current. We realized a cross-thoracic recording by placing the ground contact (acting as gate) on the chest and the OECT on the forearm as shown in Figure 3a. In order to measure in a transistor regime where transconductance is high and leakage current sufficiently low, we set the transistor channel to a potential of $V_{S G}=0.5 \mathrm{~V}$ with respect to the grounded body while monitoring the drain current at $V_{S D}=-0.3 \mathrm{~V}$. The resulting 
current trace (red line, Fig 3b) contains the typical spikes of the heart beat with an amplitude of $\sim 100 \mathrm{nA}$ in comparison to spikes of $500 \mu \mathrm{V}$ as measured with the traditional potentiometric recording (black line). Although the measurements were done with ordinary lab equipment, the signal to noise ratio of the bioresorbable transistor is already comparable to the recording based on the traditional Faradaic electrodes. A closer look to an isolated OECT signal (Figure 3c) and comparison to an idealized ECG signal as found in cardiology textbooks shows that its quality is sufficient to extract some fine structure which allows for the characterization of timings between the different sub-waves.

To conclude, we demonstrated conformable and transparent transistor devices fabricated on a fully resorbable bioscaffold. The device fabrication relies on a simple process which allows patterning of active areas of the conductive polymer PEDOT:PSS contacted by gold electrodes on the bioscaffold. The resulting structure combines biocompatibility and biodegradability with excellent electronic properties for fast and sensible potentiometric sensing in aqueous conditions. The fast ion-to-electron exchange which is crucial for bioelectric interfaces results from the intrinsic materials properties of the PEDOT:PSS layer. By optimizing the layer thickness and the channel geometry we achieve the sensing of signals down to a few tens of micro-volts at timescales of a few milliseconds. As a medically relevant bioelectric recording device, we applied the transistor in electrocardiography and show a signal-to-noise ratio that is comparable to the one of standard Faradaic electrodes. These results highlight the feasibility of realizing simple organic bioelectric interfaces on implantable bioscaffolds which would allow the recording of signals from muscular or nervous tissue to monitor health state, or which could provide electrical stimulation to influence the tissue's activity. Resorption of the bioscaffold leads to a limited lifetime of the implant in the body and the removal of the devices from the regenerated tissue can be avoided in future applications. 
Experimental Section: PLGA (molecular weight: 66-107 kDa) was bought from Sigma-Aldrich (P1941); Neutral pH PEDOT:PSS suspension $\left(\right.$ Clevios $^{\mathrm{TM}}$ Jet N V2) with added $0.2 \%$ Silquest (3glycidoxypropyltrimethoxysilane) and 5\% DMSO was used. Electrical measurements and ECG recordings were performed with an Agilent B2912A. For ECG recordings, contact pads on PLGA film $\left(1 \mathrm{~mm}^{2}\right)$ were connected to $10 \mathrm{~cm}$ long copper cables by using adhesive scotch tape (3M). Gold contacts were further shielded by Kapton tape. Details on ECG recording are reported in Supp. Inf..

\section{Supporting Information}

Supporting Information is available from the Wiley Online Library or from the author.

\section{Acknowledgments}

We thank Dr. Giulia Foschi and Silvia Tortorella for their help in preparing PLGA scaffolds, and Dr. Mauro Murgia for Au contacts. This work was funded by the EU $7^{\text {th }}$ Framework Programme [FP7/2007-2013] under Grant Agreement No. 280772, Implantable Organic Nanoelectronics (iONE-FP7) project.

Received: ((will be filled in by the editorial staff))

Revised: ((will be filled in by the editorial staff))

Published online: ((will be filled in by the editorial staff))

[1] K. Famm, B. Litt, J. K. Tracey, E. S. Boyden, M. Slaoui, Nature 2013, 496, 159.

[2] E. Delivopoulos, D. J. Chew, I. R. Minev, J. W. Fawcett, S. P. Lacour, Lab Chip 2012, 12, 2540.

[3] R. Langer, D. A Tirrell, Nature 2004, 428, 487.

[4] K. Franze, P. A Janmey, J. Guck, Annu. Rev. Biomed. Eng. 2013, 15, 227.

[5] L. S. Nair, C. T. Laurencin, Prog. Polym. Sci. 2007, 32, 762.

[6] D.-H. Kim, J. Viventi, J. J. Amsden, J. Xiao, L. Vigeland, Y.-S. Kim, J. A. Blanco, B. Panilaitis, E. S. Frechette, D. Contreras, D. L. Kaplan, F. G. Omenetto, Y. Huang, K.-C. Hwang, M. R. Zakin, B. Litt, J. A. Rogers, Nat. Mater. 2010, 9, 511.

[7] D. K. Gilding, A. M. Reed, Polymer 1979, 20, 1459.

[8] L. Lu, C. A. Garcia, A. G. Mikos, J. Biomed. Mater. Res. 1999, 46, 236.

[9] C. J. Bettinger, Z. Bao, Adv. Mater. 2010, 22, 651. 
[10] S.-W. Hwang, H. Tao, D.-H. Kim, H. Cheng, J.-K. Song, E. Rill, M. A. Brenckle, B. Panilaitis, S. M. Won, Y.-S. Kim, Y. M. Song, K. J. Yu, A. Ameen, R. Li, Y. Su, M. Yang, D. L. Kaplan, M. R. Zakin, M. J. Slepian, Y. Huang, F. G. Omenetto, J. A. Rogers, Science 2012, 337, 1640.

[11] M. Irimia-Vladu, P. A. Troshin, M. Reisinger, L. Shmygleva, Y. Kanbur, G. Schwabegger, M. Bodea, R. Schwödiauer, A. Mumyatov, J. W. Fergus, V. F. Razumov, H. Sitter, N. S. Sariciftci, S. Bauer, Adv. Funct. Mater. 2010, 20, 4069.

[12] N. A. Kotov, J. O. Winter, I. P. Clements, E. Jan, B. P. Timko, S. Campidelli, S. Pathak, A. Mazzatenta, C. M. Lieber, M. Prato, R. V. Bellamkonda, G. A. Silva, N. W. S. Kam, F. Patolsky, L. Ballerini, Adv. Mater. 2009, 21, 3970.

[13] M. Kaltenbrunner, T. Sekitani, J. Reeder, T. Yokota, K. Kuribara, T. Tokuhara, M. Drack, R. Schwödiauer, I. Graz, S. Bauer-Gogonea, S. Bauer, T. Someya, Nature 2013, 499, 458.

[14] A. N. Sokolov, C. J. Bettinger, Z. Bao, Acc. Chem. Res. 2012, 45, 361.

[15] T. Cramer, A. Campana, F. Leonardi, S. Casalini, A. Kyndiah, M. Murgia, F. Biscarini, J. Mater. Chem. B 2013, 1, 3728.

[16] O. Knopfmacher, M. L. Hammock, A. L. Appleton, G. Schwartz, J. Mei, T. Lei, J. Pei, Z. Bao, Nat. Commun. 2014, 5, 2954.

[17] P. Bergveld, J. Wiersma, H. Meertens, S. Member, IEEE T. Bio-Med Eng 1976, 23, 136.

[18] P. Fromherz, A. Offenhausser, T. Vetter, and J. Weis, Science 1991, 252, 1290.

[19] D. Khodagholy, T. Doublet, P. Quilichini, M. Gurfinkel, P. Leleux, A. Ghestem, E. Ismailova, T. Hervé, S. Sanaur, C. Bernard, G. G. Malliaras, Nat. Commun. 2013, 4, 1575.

[20] T. Cramer, B. Chelli, M. Murgia, M. Barbalinardo, E. Bystrenova, D. M. de Leeuw, F. Biscarini, Phys. Chem. Chem. Phys. 2013, 15, 3897.

[21] E. Stavrinidou, P. Leleux, H. Rajaona, D. Khodagholy, J. Rivnay, M. Lindau, S. Sanaur, G. G. Malliaras, Adv. Mater. 2013, 25, 4488.

[22] G. Tarabella, F. Mahvash Mohammadi, N. Coppedè, F. Barbero, S. Iannotta, C. Santato, F. Cicoira, Chem. Sci. 2013, 4, 1395.

[23] P. Lin, F. Yan, J. Yu, H. L. W. Chan, M. Yang, Adv. Mater. 2010, 22, 3655.

[24] A. Campana, T. Cramer, P. Greco, G. Foschi, M. Murgia, F. Biscarini, Appl. Phys. Lett. 2013, 103, 073302.

[25] J. Y. Yoo, J. M. Kim, K. S. Seo, Y. K. Jeong, H. B. Lee, G. Khang, Biomed. Mater. Eng. 2005, 15, 279.

[26] D. Khodagholy, J. Rivnay, M. Sessolo, M. Gurfinkel, P. Leleux, L. H. Jimison, E. Stavrinidou, T. Herve, S. Sanaur, R. M. Owens, G. G. Malliaras, Nat. Commun. 2013, 4, 2133. 
[27] B. D. Nilsson, M. Chen, T. Kugler, T. Remonen, M. Armgarth, M. Berggren, Adv. Mater. 2002, 14, 51 .

[28] Y. M. Chi, S. Member, T. Jung, S. Member, G. Cauwenberghs, IEEE Rev Biomed Eng 2010, 3, 106.

[29] G. Tarabella, C. Santato, S. Y. Yang, S. Iannotta, G. G. Malliaras, F. Cicoira, Appl. Phys. Lett. 2010, 97, 123304. 

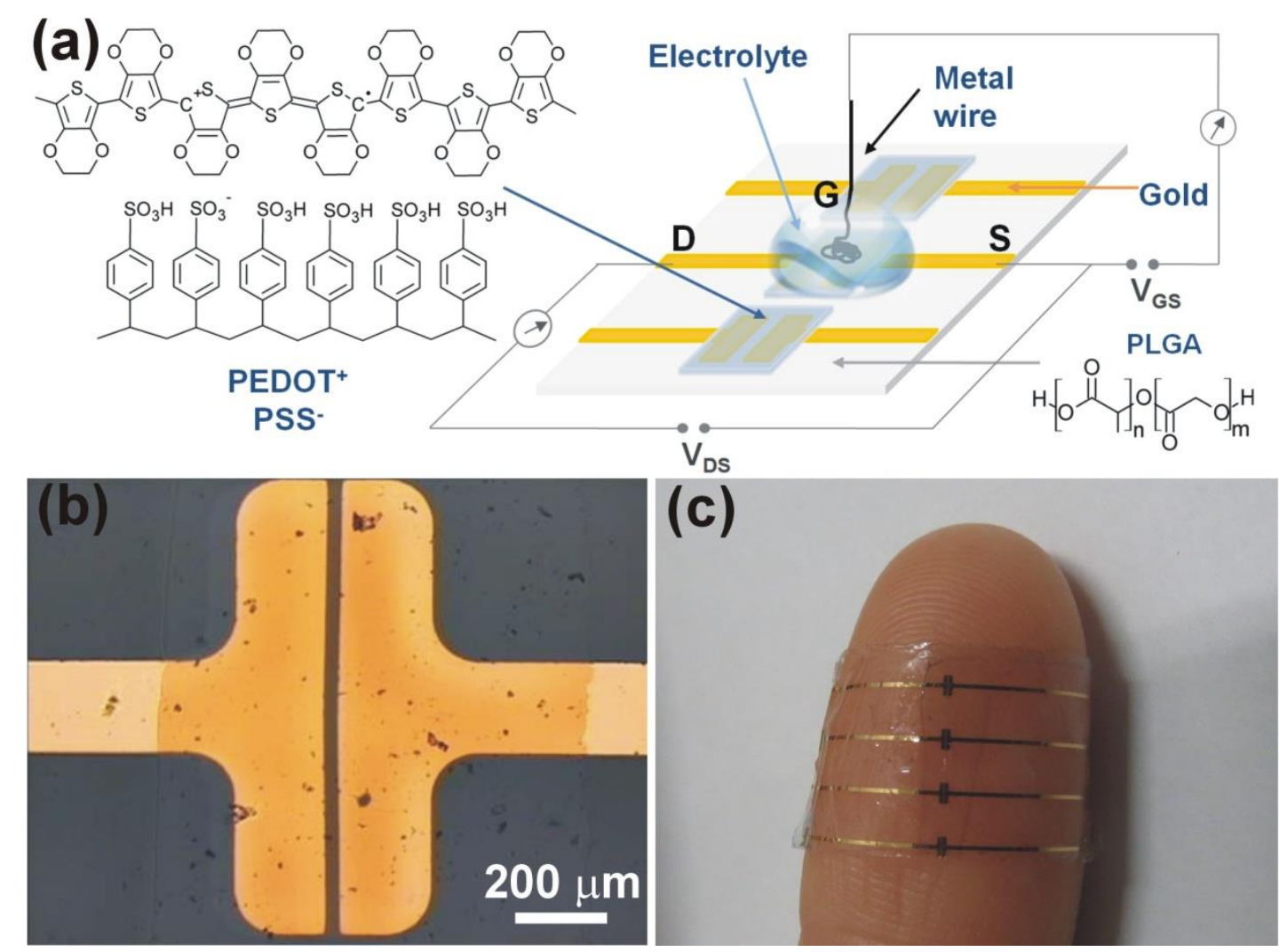

Figure 1. (a) Organic electrochemical transistor (OECT) structure and materials. PEDOT:PSS is patterned as the active layer on the bioresorbable PLGA thin film and contacted by gold source and drain electrodes. The structure is gated by the electronic potential applied to the aqueous electrolyte via a metal wire. (b) Optical microscopy image of an individual transistor. The darker color of the electrodes indicated the area on which PEDOT:PSS is present. (c) Photograph of the device showing its transparency and adaptability when attached to human skin. 

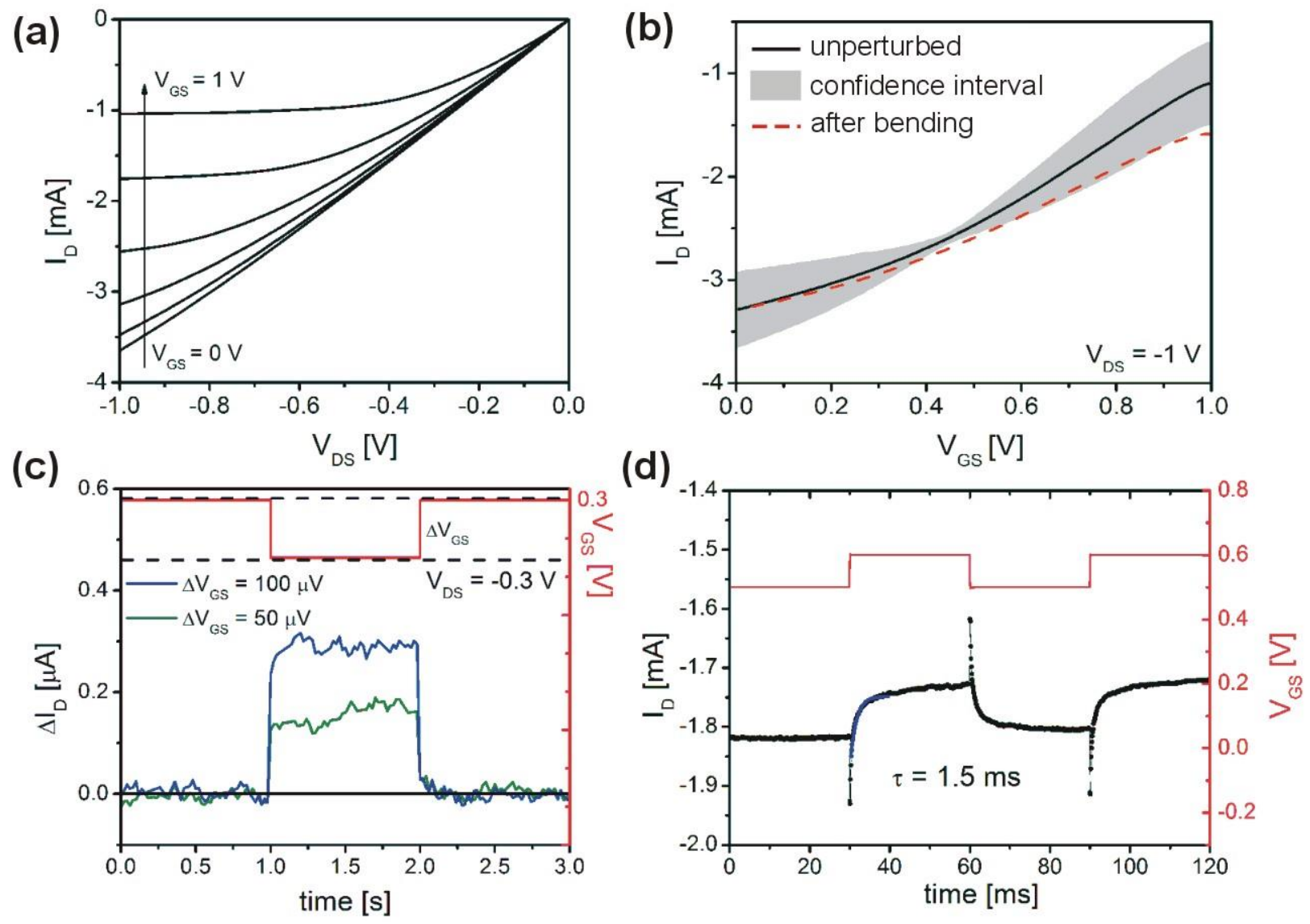

Figure 2. OECT characterization and potentiometric sensing in aqueous electrolyte. (a) Output characteristics. (b) Transfer characteristics of unperturbed devices (black with the grey area indicating the experimental variation) and after bending at a curvature radius of $80 \mu \mathrm{m}$. (c) Drain current variation as a consequence to potential changes as small as $50 \mu \mathrm{V}$ applied to the gate. (d) Fast response ( $\tau=1.5 \mathrm{~ms}$ ) of the drain current $\left(V_{D S}=-0.5 \mathrm{~V}\right)$ to a gate voltage variation of $0.1 \mathrm{~V}$. 

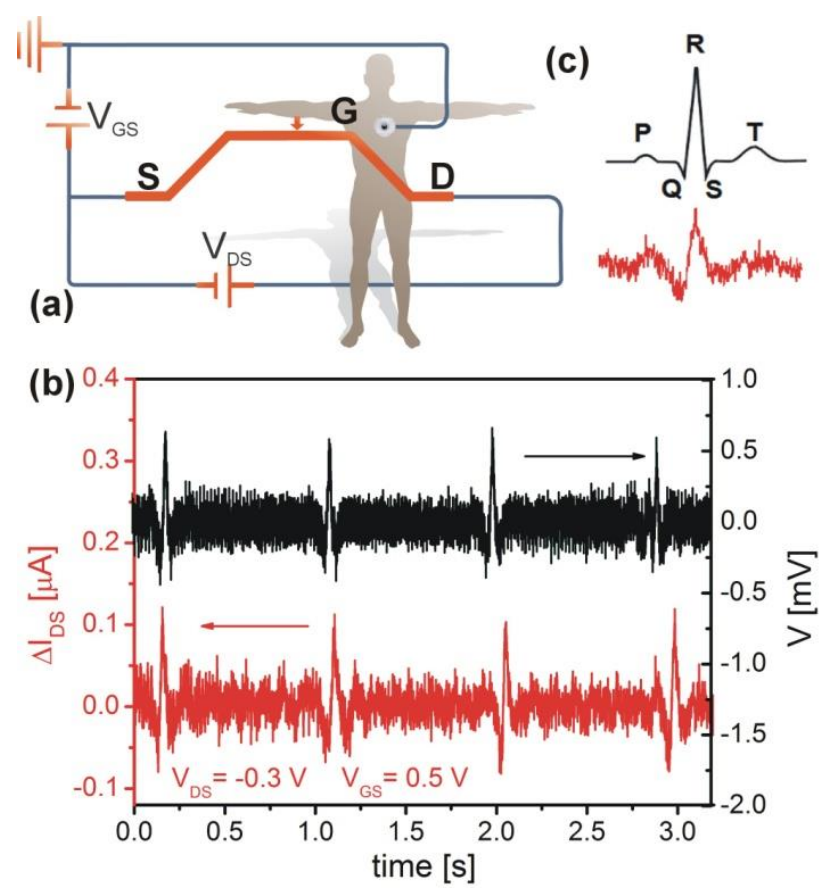

Figure 3. ECG recording with a bioresorbable OECT operated in direct contact with the skin. (a) Wiring diagram of the experiment. (b) Measured drain current trace (red) as obtained during ECG recording $\left(V_{S G}=\right.$ $0.5 \mathrm{~V}, V_{S D}=-0.3 \mathrm{~V}$ ) and comparison to a normal potentiometric recording with standard disposable leads (black). (c) Enlarged transistor ECG trace of a single cardiac cycle and comparison to a schematic textbook example. 